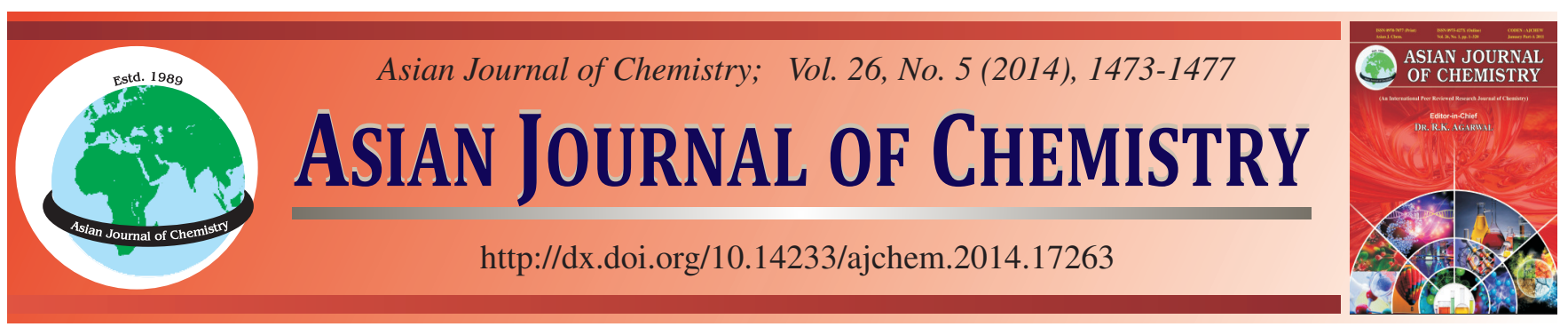

\title{
Mineralization of Chinese Medicine Wastewater and Power Generation Using an Improved Salt Bridge Microbial Fuel Cell $\dagger$
}

\author{
Jianping Cheng ${ }^{1}$, Shaohua Chen $^{2}$, Zhiguo Tang ${ }^{1}$, Ting Cheng ${ }^{2}$ and Jiaquan Wang ${ }^{2, *}$
}

${ }^{1}$ School of Mechanical and Automotive Engineering, Hefei University of Technology, Hefei 230009, P.R. China

${ }^{2}$ School of Resource and Environment Engineering, Hefei University of Technology, Hefei 230009, P.R. China

*Corresponding author: E-mail: jiaquan.wang@163.com

Published online: 1 March 2014;

\begin{abstract}
High cost is still a problem facing the development of microbial fuel cells. Dual-chambered microbial fuel cells were constructed whose salt bridges substituted for proton membranes and were modified. The microbial fuel cell reactors were designed to test the potential for pollutant degradation and generated electricity using glucose and Chinese medicine wastewater. Experimental results showed that the salt walls structure microbial fuel cell was the best in microbial fuel cells of three kinds of structure in generated electricity and removal of chemical oxygen demand (COD). Then as a reactor by the microbial fuel cell of low resistance, the maximal power density was obtained was $100 \mathrm{~mW} / \mathrm{m}^{2}$, the colour removal efficiency was 97 and $70 \%$, the value of BOD $_{5} / \mathrm{COD}$ was 0.14 up to 0.26 and 0.14 up to 0.20 in closed circuit and open circuit, respectively. Results indicated that the microbial fuel cell of low resistance of salt wall had a better performance.
\end{abstract}

Keywords: Microbial fuel cell, Salt bridge, Chinese medicine wastewater.

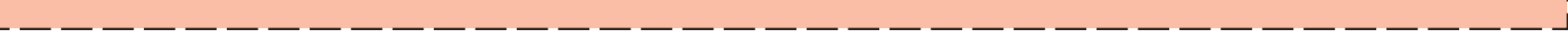

\section{INTRODUCTION}

Microbial fuel cells (MFCs) are devices in which bacteria create electrical power by oxidizing simple compounds such as glucose or complex organic matter in wastewater ${ }^{1,2}$. Microbial fuel cell technology can steady generate energy from sludge under no addition of redox mediators ${ }^{3,4}$, it was realized that sewage biologically produced electricity. This finding provides a new way of thinking for the low-cost organic wastewater treatment ${ }^{5,6}$. But as a new sewage treatment technology in terms of energy output or cost, compared with mature anaerobic biological treatment technology, there is a significant gap ${ }^{7}$. In particular, the cost is one of the key issues that limit the scaleup application of $\mathrm{MFC}^{8}$.

The traditional dual-chamber MFC ordinarily consists of two chambers, one anode (anaerobic) and the other cathode (aerobic). In the anode chamber, substrate is oxidized by bacteria and the electrons transferred to the anode either by an exogenous electron carrier, or mediator. The anode chamber is connected internally to the cathode chamber by a proton-conducting material and externally by a wire that completes the circuit. In the cathode chamber, electrons that pass along the circuit combine with protons and oxygen to form water. Proton conductive materials in a MFC should ideally be able to inhibit the transfer of other materials such as the fuel or the electron acceptor while conducting protons to the cathode at high efficiency. Materials used in MFCs include fluoropolymercontaining cation exchange materials such as Nafion ${ }^{\mathrm{TM}}$ and even systems without a membrane ${ }^{9,10}$. Nafion ${ }^{\text {TM }}$ is the most intensively studied fuel cell membrane as it provides high ionic conductivity. The main limitations of Nafion ${ }^{\mathrm{TM}}$ are its high cost, restricted temperature range and oxygen permeability. The presence of the membrane greatly improved the cost of the MFC, the salt bridge replaced the proton exchange membrane which greatly reduces the cost of the $\mathrm{MFC}^{-11}$. However, as proton circulation channel, how to reduce internal resistance of salt bridge is the problem of this type of the MFC structure facing ${ }^{12}$.

In this study, dual-chamber salt bridge MFCs were designed and their structures of salt bridge were improved to reduce the internal resistance and then an improved MFC was used to deal with the high concentration of Chinese medicine wastewater (CMW) compared with the conventional anaerobic biological treatment technology.

†Presented at The 7th International Conference on Multi-functional Materials and Applications, held on 22-24 November 2013, Anhui University of Science \& Technology, Huainan, Anhui Province, P.R. China 


\section{EXPERIMENTAL}

Culture and medium: Glucose $3 \mathrm{~g} / \mathrm{L}$ (COD of $3000 \mathrm{mg}$ / L) or medicine wastewater dissolved in the inorganic salt is the carbon source of microorganisms of the anode chamber. The inorganic salt is a main component: $\mathrm{KCl} 0.13 \mathrm{~g} / \mathrm{L}, \mathrm{NH}_{4} \mathrm{Cl}$ 0.31 and $12.5 \mathrm{~g} / \mathrm{L}$ and vitamins $5 \mathrm{~g} / \mathrm{L} . \mathrm{K}_{2} \mathrm{HPO}_{4} 6.571 \mathrm{~g} / \mathrm{L}$ and $\mathrm{KH}_{2} \mathrm{PO}_{4} 2.883 \mathrm{~g} / \mathrm{L}$ configured as a phosphate buffer solution are added to the cathode chamber and the anode chamber.

The anode chamber was inoculated activated sludge taken from Hefei Wang Xiao-ying sewage treatment plant. Carbon resource dissolved in inorganic salt before entering the anode chamber, which leads to 15-20 min of nitrogen to remove dissolved oxygen and then quickly fitted to the test reactor and sealed to maintain an anaerobic environment. Under ambient conditions, the MFC closed circulation system culture a week, when the output voltage is less than the MFC $20 \mathrm{mV}$ or less, that the end of the trial.

The Chinese medicine wastewater used for Anhui in pharmaceutical production plant wastewater, pharmaceutical company mainly produces pure Chinese medicine off the blood flow, the major organic ingredients of this preparation as flavonoids and saponins. COD concentration of $28,000 \mathrm{mg} / \mathrm{L}, \mathrm{BOD}_{5}$ $2640 \mathrm{mg} / \mathrm{L}, \mathrm{pH} 5.34$, the colour of 10000 times (dilution factor method).

The initial $\mathrm{pH}$ of all solutions was adjusted to 7 and all MFCs were operated in a temperature-controlled room at $25^{\circ} \mathrm{C}$. In some tests, $\mathrm{HCl}(0.5 \mathrm{~g} / \mathrm{L}, \mathrm{Calbiochem})$ was added to chemically scavenge dissolved oxygen. $\mathrm{HCl}$ reacts with dissolved oxygen to form cystine, a disulfide-bonded dimer.

Microbial fuel cell construction and operation: The two-chambered MFCs were used throughout the study. There are three kinds of MFCs. The structure of MFC-1 is two-bottles salt bridge which can split. The anode chamber and the cathode chamber, respectively are made of a $400 \mathrm{~mL}$ conical flask and beaker. An optional salt bridge connecting the dual chamber is a diameter of $5 \mathrm{~mm}$, length $500 \mathrm{~mm}$ plastic tube is filled with agar-KCl semi-solidas Fig. 1; Figs. 1 and 2 are used in this experiment salt bridge structure integral and split the salt bridge structure. Fig. 2 is the overall structure of the MFC-1 dual chamber, a salt bridge connecting the two-chamber structure of the salt bridge perforated plate, like a wall, parallel channels 100 uniform in cross section, diameter of $3 \mathrm{~mm}$, length $10 \mathrm{~mm}$, the anode and cathode compartments of the glass vessel of $250 \mathrm{~mL}$, respectively; Fig. 3 by a plurality of parallel channels MFC-3 becomes the complete cylindrical passage communicating, diameter of $30 \mathrm{~mm}$, length $50 \mathrm{~mm}$, removable, respectively, the anode and cathode chamber of $250 \mathrm{~mL}$ plexiglass container. Two kinds of structure of MFC anode material were cylindrical brush, diameter $3 \mathrm{~mm}$, length $5 \mathrm{~mm}$. PRB monolithic structure similar to the structure MFC-1 and MFC-2 applied to in situ remediation of contaminated groundwater and split structure for the requirements of the anode chamber and the cathode chamber of MFC-3 requires far more competent such as groundwater treatment.

All salt bridge were prepared as following: 4G agar was placed in $200 \mathrm{~mL}$ of distilled water in the beaker, to when agar is completely dissolved by electric heating, then $60 \mathrm{~g} \mathrm{KCl}$ were added again and continue stirring and heating until the

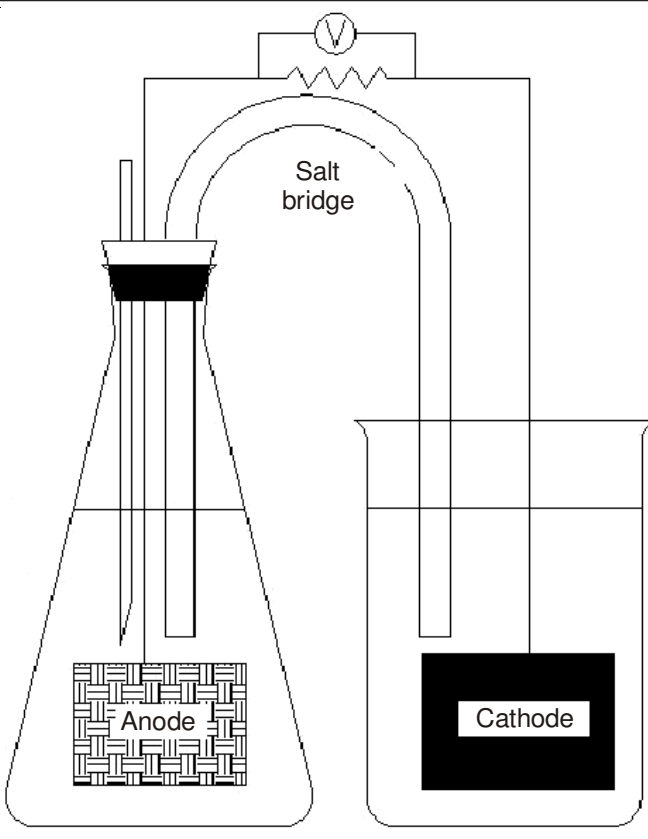

Fig. 1. Dual-bottle salt bridge structure of MFC-1

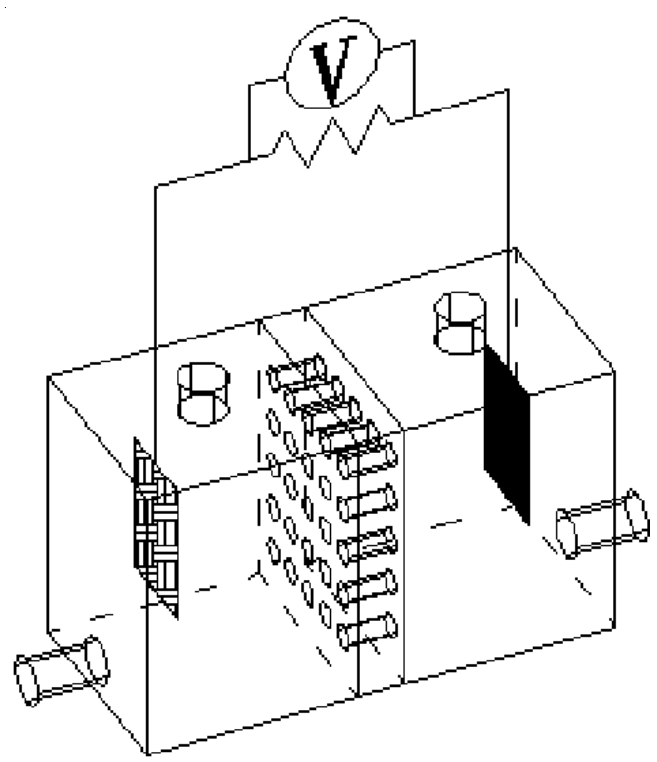

Fig. 2. Porous sieve structure of MFC-2

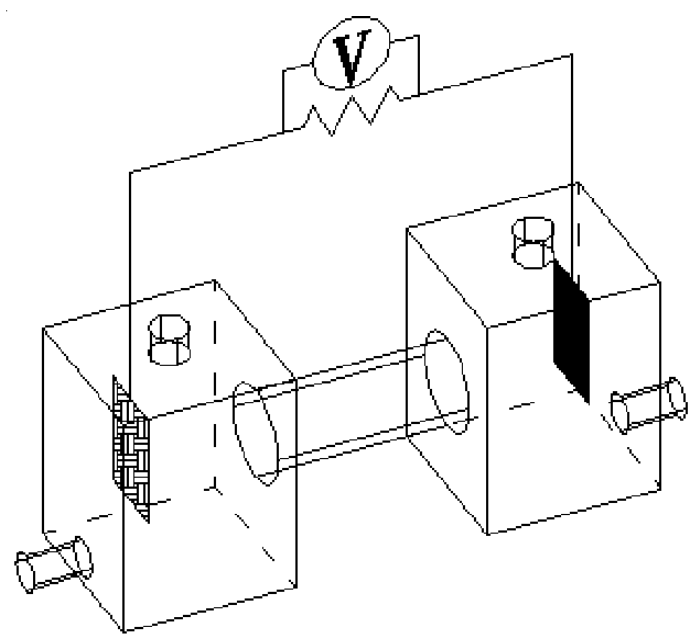

Fig. 3. Salt walls structure of MFC-3 
$\mathrm{KCl}$ is completely dissolved, syringe while hot plastic hose mixed solution was injected into the appropriate length prepared in action, into coherent quickly, in order to prevent bubble containing salt bridge, agar after cooling into the gel, with a blade to scrape off the excess agar, standby vessel immersed in distilled water.

The material of anode and cathode materials of MFCs were titanium $\beta-\mathrm{PbO}_{2}$ and carbon cloth which dimensions are $4 \mathrm{~cm} \times 4 \mathrm{~cm}$ in the paper and preparation of the titaniumbased $\beta-\mathrm{PbO}_{2}$ electrode ${ }^{13}$.

Analyses and calculations: The voltage generated in MFCs was measured by using a voltammeter with an external resistor $(1000 \Omega)$ connected across the electrodes and recorded after regular time intervals. The voltage values were recorded only after a steady state. Current (i) was calculated using relationship, $\mathrm{i}=\mathrm{V} / \mathrm{R}$, where $\mathrm{V}$ is the voltage and $\mathrm{R}$ is resistance. The maximum power generated was calculated using the following formula: $P_{\max }=V_{\max } \times I$, (1) where $V$ is the maximum open circuit voltage (OCV) and I is the corresponding current. Power density $\left(\mathrm{mW} / \mathrm{m}^{2}\right)$ was calculated by dividing the obtained power with the surface area $\left(\mathrm{m}^{2}\right)$ of the anode, respectively. Chemical oxygen demand (COD) of glucose and were measured using standard methods. Colour removal of CMW was determined by dilution analysis. The biodegradability was done by $\mathrm{BOD}_{5} / \mathrm{COD}$.

\section{RESULTS AND DISCUSSION}

The power density increased with time of operation after start-up. Constant COD removal efficiency and stable voltage generation were considered as indicators to assess the stabilized performance of the MFC and subsequently polarization study was done. Stable voltage and COD removal was observed after 16 days of operation in MFC-1, MFC-3 and MFC-2. The performance of MFC with respect to power generation and substrate removal was found to be dependent on the applied pre-treatment to the sludge inoculums.

\section{Comparison of three kinds of MFCs}

Power generation: As shown in Fig. 1, three kinds of MFC-1, MFC-2 and MFC-3 all can generate electricity. production capacity of MFC- 1 is the weakest, the maximum output power density is only $100 \mathrm{~mW} / \mathrm{m}^{2}$ and maintain the most short time, it is in $100 \mathrm{~h}$, this is because the MFC- 1 salt bridge of small diameter, length, mass transfer resistance, internal resistance of battery increases with time, output power weakened. The electricity production capacity of MFC-2 and MFC-3 are stronger, the highest output power density can reach 250 $\mathrm{mW} / \mathrm{m}^{2}$, stable time is longer than MFC-1. This is because their salt bridge cross section area and length were beneficial to mass transfer than MFC-1, so that the output power of the battery. But MFC-2 compared with MFC-3, MFC-2 as the salt bridge length is less than MFC-3, is conducive to mass transfer, output power of the battery quickly rose to $300 \mathrm{of} \mathrm{mW} / \mathrm{m}^{2}$, more than MFC-3 of the maximum output power density of $250 \mathrm{~mW} / \mathrm{m}^{2}$, but MFC-2 in the output power density higher maintenance time is less than MFC-3 in the output power density high maintenance time, which may be attributable to the salt bridge section size, the cross-sectional area of the salt bridge MFC-3 is greater than MFC-2, more conducive to mass transfer.
COD concentration of the three MFCs in the anode solution: The COD concentration in the anode solution changes over time shown in Fig. 5. As shown in Fig. 5, when the measured voltage is less than $20 \mathrm{~mW}$, the detection to MFC- 1 anode chamber solution COD is only decreased by $60 \%$; MFC-2 and MFC-3 anode chamber in the solution of the COD dropped by more than $90 \%$, but degradation rate is fastest in the MFC2 , the time of generated electricity to maintain is longest in the MFC-3.

The degradation of COD glucose anode chamber structure of three kinds of organic compounds in the MFC results are shown in Figs. 4 and 5. It can be seen from Figs. 4 and 5, the MFC-1 in the battery power after first cycles, organic matter measured anode chamber of COD decreased from 3000-986 $\mathrm{mg} / \mathrm{L}$, the removal rate of $60 \%$ and MFC- 2 on battery power after first cycles, organic matter measured anode chamber of COD decreased from 3000 to $212 \mathrm{mg} / \mathrm{L}$, the removal rate reached $93 \%$, MFC-3 battery power after first cycles, organic matter measured anode chamber of COD decreased from 3000 to $226 \mathrm{mg} / \mathrm{L}$, the removal rate reached $92 \%$, wherein the anode of organic compounds in MFC-2 COD removal rate was higher than MFC-3. The results with the battery output power is consistent.

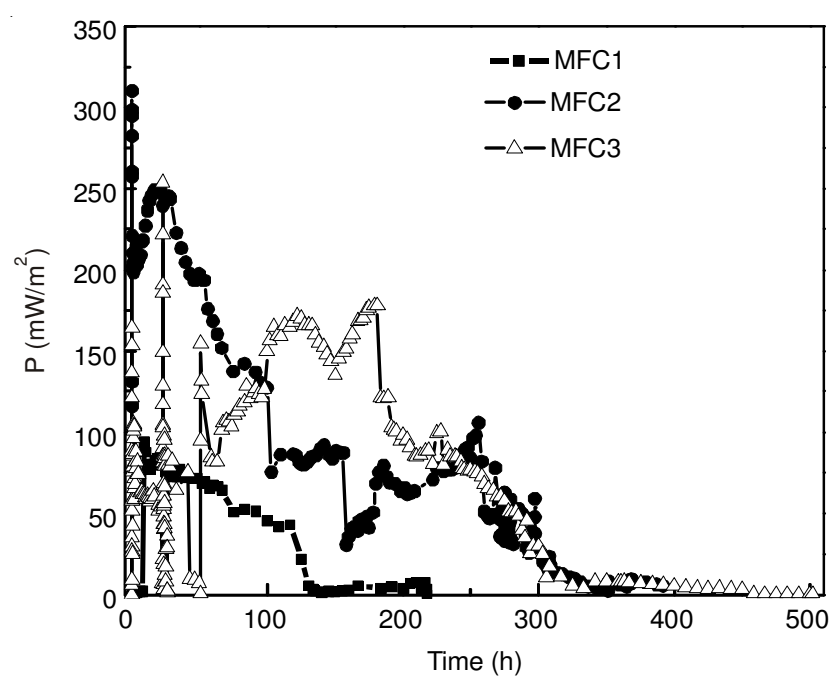

Fig. 4. Variation of power density with times in MFCs

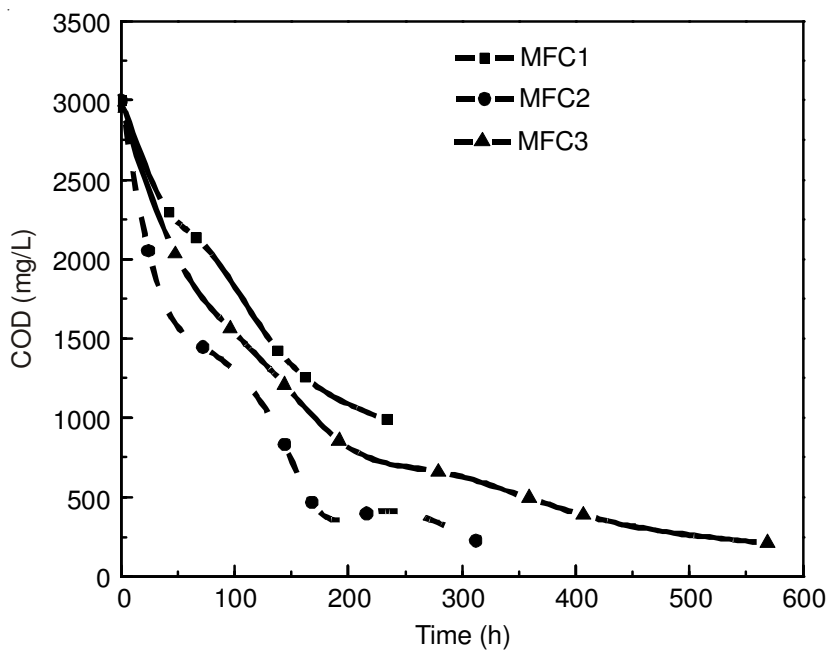

Fig. 5. COD removal in the anode solution in MFCs 
Two salt bridges MFCs in promoting the degradation of organic matter: In order to compare two kinds of improved salt bridge structure MFC-2, MFC-3 and conventional anaerobic reactor on the anode of organic matter degradation effect, the running result of MFC circuit and open circuit in two ways in the same conditions, as shown in Fig. 6. Fig. 6 is the result of MFC-2 and MFC-3, respectively in the closed and open experiment measured COD concentration of glucose in the anode chamber. Fig. 6 showed the same treatment time, glucose degradation effect of MFC-2 and MFC-3 in the closed state than the degradation in the open state effect is more thorough, such as MFC-3 after $522 \mathrm{~h}$ treatment, open anode system under condition of COD removal rate reached $65 \%$ and the anode system closed under condition of COD removal the rate reached $90 \%$. The results showed that two kinds of structure of MFC could promote the anaerobic microbial anode system for the decomposition of glucose, improve the removal effect of COD anode system.

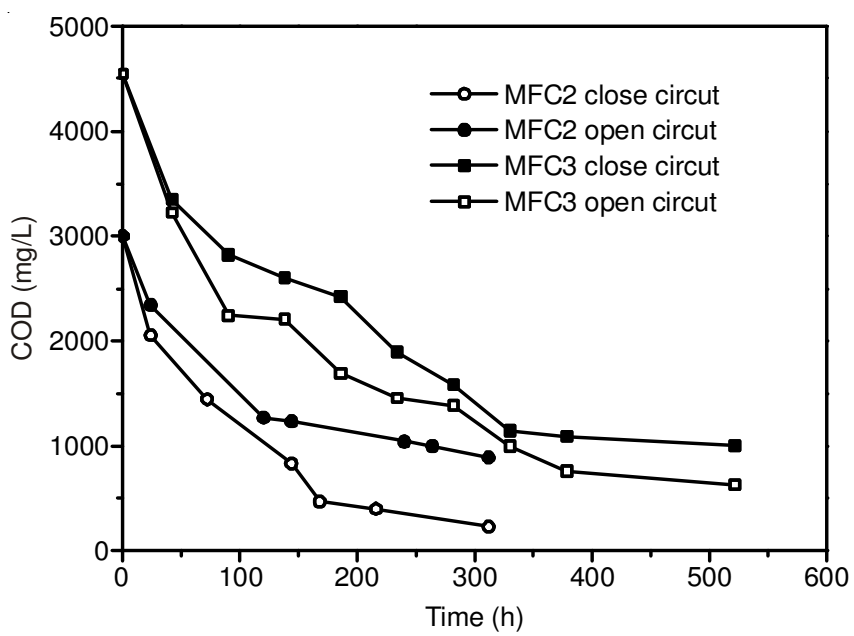

Fig. 6. COD concentration in a closed-circuit and open-circuit test after MFC-2 and MFC-3 measured anode chamber

Based on the above results, the MFC reactor in three kinds of comparison, through the degradation of electricity production, anode organic substance and salt bridge stability time, the reactor will order: MFC-3 is superior to MFC-2 is better than MFC-1, so in the following experiment to choose MFC-3 as the reactor for the following experiment.

\section{Mineralization of Chinese medicine wastewater and power generation}

Power generation: The actual characteristics of traditional Chinese medicine wastewater is a high concentration of organic pollutants, highly coloured, using biological methods usually, this experiment with traditional Chinese medicine wastewater diluted as anode carbon source, with small resistance wall structure of electricity generation a better performance than the MFC as the reactor, the effects of MFC on electrical production and traditional Chinese medicine wastewater the degradation effect.

Figs. 7 and 8 showed that the diluted medicine wastewater as carbon source for the MFC-3 anode chamber, the output power density and COD concentration in the anode changed with time. As can be seen from Fig. 7, MFC-3 has not been

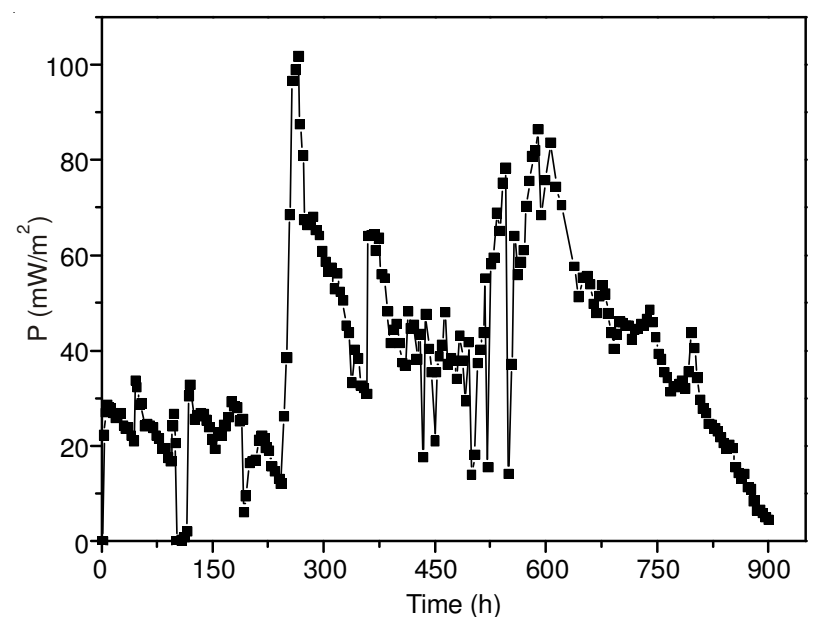

Fig. 7. Power densities of medicine wastewater in the MFC-3

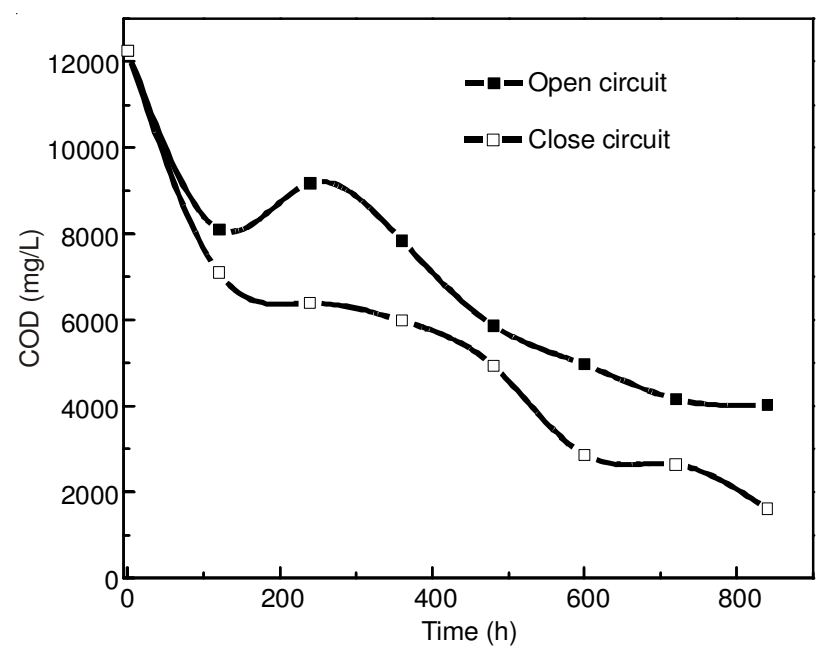

Fig. 8. COD removal of medicine wastewater in the MFC-3

inoculated sludge medicine wastewater acclimation, therefore, the electricity generated is rare in the initial $200 \mathrm{~h}$, but with the adaptation of microbial populations in the anode of this medicine wastewater energy generated increases rapidly and to maintain at $40 \mathrm{~mW} / \mathrm{m}^{2}$ the level within the $400 \mathrm{~h}$, the organic decomposition until after $650 \mathrm{~h}$, the anode chamber, $\mathrm{pH}$ reduced to result in the internal environment gradually lose balance inappropriate microorganisms continue to grow, so that the energy output of the battery decrease. As can be seen from Fig. 8, COD removal of medicine wastewater in the MFC-3 in close circuit is higher than in open circuit, this proved MFC can strengthen the degradation process.

Degradation of CWM using small resistance MFC with salt wall structure: Table-1 showed that colour removal efficiencies and the value of $\mathrm{BOD}_{5} / \mathrm{COD}$ changing as the function of time between a close circuit and an open circuit. The results indicated accelerated colour removal of Chinese medicine wastewater in the double-chamber MFCs as compared to a traditional anaerobic reactor in a circuit. The colour was removed almost completely after a circle in the MFCs, while $70 \%$ was removed for the traditional anaerobic reactor. The results in this study might be attributed to the presence of the anode, which increases the metabolic rate of anaerobic bacteria with sufficient anaerobic terminal electron acceptors. This could result in faster conversion of the co-substrate to produce more 


\begin{tabular}{ccccccc}
\hline \multicolumn{7}{c}{ COLOUR REMOVAL AND BOD $/$ COD CHANGE OF CMW UNDER TWO DIFFERENT CIRCUITS } \\
\hline \multirow{2}{*}{$\begin{array}{c}\text { Time } \\
\text { (h) }\end{array}$} & Colour (times) & Colour removal efficiencies $(\%)$ & BOD $_{5} /$ COD & Colour (times) & Colour removal efficiencies $(\%)$ & BOD $_{5} /$ COD \\
\hline 0 & 3300 & 0 & 0.14 & 3300 & 0 & 0.14 \\
1,000 & 100 & 97 & 0.26 & 1000 & 70 & 0.20 \\
\hline
\end{tabular}

electrons, realizing an indirect accelerated colour removal of Chinese medicine wastewater. In the open circuit test, only biological processes responded for colour removal while in the close circuit test physiochemical processes may provide other means for colour removal ${ }^{14}$. Therefore, the physio-chemical processes may contribute to the extra $27 \%$ colour removal in the close circuit test, possibly including electricity generation and volatilization caused of current. At the same time, to further elucidate the wastewater removal, the experiment also measured the value of $\mathrm{BOD}_{5} / \mathrm{COD}$, the result indicated the value of $\mathrm{BOD}_{5} / \mathrm{COD}$ increased after MFC operation, ranging from 14 to $26 \%$. These data further testified the biodegradability of wastewater can be mainly enhanced after the MFCs operation ${ }^{15}$.

\section{Conclusion}

Dual-chamber MFC salt bridge instead of the proton exchange membrane, through changes the MFC in salt bridge structure, the MFC internal resistance and lower cost and performance of the electricity production, anode degradation of organic matter is more thoroughly, of which the best small resistance effect of salt wall structure MFC. Microbial fuel cell effect of the salt wall structure of the large resistance, followed by the worst MFC effect of the salt bridge structure. That bridge is used for MFC is feasible and effective and make MFC for the actual sewage treatment becomes possible. It greatly reduces the design and the construction difficulty in practical applications. There are broad prospects for development in the sewage and production capacity. This study demonstrated the feasibility of using the small resistance proton-wall MFC using Chinese medicine wastewater as substrates to generate electricity and simultaneously treat Chinese medicine wastewater with high removals of COD and colour, providing an attractive alternative to reduce the cost of wastewater treatment and generate electricity. Although additional studies are needed to scale up and optimize the process. Over the entire experiment, the maximum power density of $90 \mathrm{~mW} / \mathrm{m}^{2}$ was obtained. Maximum removal efficiencies of colour and the value of $\mathrm{BOD}_{5} /$ COD were 97 and $0.26 \%$, respectively in the close circuit, comparing with 70 and $0.20 \%$ in the open circuit after 1,000 $\mathrm{h}$ operation. It means the biodegradability of wastewater can be mainly enhanced after MFCs operation.

\section{ACKNOWLEDGEMENTS}

This work was financially supported by the National Science Foundation of China (No.41372246) and Young Teachers Fund Project of Hefei University of Technology.

\section{REFERENCES}

1. B.A. Jan, Microb. Biotechnol., 1, 1 (2011).

2. B.E. Logan and K. Rabaey, Science, 337, 686 (2012).

3. B.E. Logan and M. Elimelech, Nature, 488, 313 (2012).

4. Z. Du, H. Li and T. Gu, Biotechnol. Adv., 25, 464 (2007).

5. L. Huang, L. Gan, N. Wang, X. Quan, B.E. Logan and G. Chen, Biotechnol. Bioeng., 109, 2211 (2012).

6. S. Cheng, D. Xing and B.E. Logan, Biosens. Bioelectron., 26, 1913 (2011).

7. S.T. Oh, J.R. Kim, G.C. Premier, T.H. Lee, C. Kim and W.T. Sloan, Biotechnol. Adv., 28, 871 (2010).

8. B.E. Logan, Appl. Microbiol. Biotechnol., 85, 1665 (2010).

9. L. Zhuang, C. Feng, S. Zhou, Y. Li and Y. Wang, Process Biochem., 45, 929 (2010).

10. Y. Zuo, S. Cheng and B.E. Logan, Environ. Sci. Technol., 42, 6967 (2008).

11. Y. Zuo, S. Cheng, D. Call and B.E. Logan, Environ. Sci. Technol., 41, 3347 (2007).

12. B. Min, S. Cheng and B.E. Logan, Water Res., 39, 1675 (2005).

13. S. Chen, J. Wang, X. Xia, C. Chen, J. Yang and Y. Zhu, Asian J. Chem., 24, 3997 (2012).

14. B. Min, J.R. Kim, S.E. Oh, J.M. Regan and B.E. Logan, Water Res., 39, 4961 (2005).

15. J. Sun, Y.- Hu, Z. Bi and Y.- Cao, Bioresour. Technol., 100, 3185 (2009). 